\section{Osteocytes, bone remodeling and parathyroid hormone}

\author{
Nabanita S. Datta \\ Department of Internal Medicine, \\ Endocrinology, Cardiovascular Research \\ Institute, Karmanos Cancer Institute, \\ Wayne State University School \\ of Medicine, Detroit, MI, USA
}

\section{Abstract}

Osteocytes, the longest living bone cells, have garnered much of the attention in the part they play in skeletal biology to orchestrate bone homeostasis. Although the multifunctional role of osteocytes in bone remodeling has been recognized, knowledge about the activity of important signaling molecules in osteocytes in this process are limited and far from being clearly depicted than osteoblast's and osteoclast's involvement. Stimulating the function of boneforming osteoblasts and controlling osteoclastic activity are the preferred pharmacological intervention for skeletal repair. A large variety of studies have been conducted in osteoblasts and osteoclasts on recombinant parathyroid hormone (PTH), an anabolic drug which proved to be effective for bone strength and clinical therapy. Studies in relation to newly functioning signaling molecules in osteocytes and PTH's mode of action is becoming an intense area of investigation suggesting further the importance of these cells in bone anabolic action. The goal of this review is to discuss briefly the function and physiological roles of osteocytes and highlight a few recent findings on the PTH's action in osteocyte signaling in health and disease. In this context this review in addition to Pubmed searches also borrows many literatures in bone and related fields from various publications.

\section{What are osteocytes}

Osteocytes (Figure 1), the star-shaped cells, are terminally differentiated cells of the osteoblast lineage. Some osteoblasts after laying down osteoid matrix harmonize its mineralization and subsequently become embedded in mineralized matrix. These cells known to be instrumental in coordinating osteoblast and osteoclast activity, and more recently has been recognized as an endocrine cell. ${ }^{1,2}$ Being ten times more abundant than osteoblasts, osteocytes have the potential to live long.,34 The loss of osteocytes from human bones is associated with the ageing process.5,6
Osteocytes contain a nucleus and a thin ring of cytoplasm. The space that an osteocyte occupies is called a lacuna (15-20 um, Latin for a pit). Osteocytes are networked to each other via long cytoplasmic extensions that occupy tiny canals called canaliculi (250-300 nm in diameter), which are used for exchange of nutrients and waste through gap junctions. ${ }^{7}$ Although osteocytes have reduced synthetic activity and, unlike osteoblasts, are not capable of mitotic division, they are actively involved in the routine turnover of bony matrix, through various mechanosensory mechanisms. These cells destroy bone through a rapid and transient (relative to osteoclasts) mechanism called osteocytic osteolysis. Studies also show that osteocytes can remove mineralized bone matrix using mechanisms like osteoclasts and by reversibly remodeling their perilacunar/canalicular matrix. ${ }^{8}$ It was suggested that osteocytic remodeling is concurrent with increase in tartrate resistant acid phosphatase activity and cathepsin $\mathrm{K}$ expression. The dynamics of the transition to osteocyte from osteoblast is being studied by manipulating gene expression in osteocytes in transgenic mouse models carrying lineage reporters for osteoblasts and osteocytes and has been reviewed by Dallas and Bonewald. ${ }^{9}$ DNA methylation as a contributor of osteoblast to osteocyte transition has also been suggested very recently. ${ }^{10}$

\section{Functions of osteocytes}

Mechanistic understanding of mechanobiology, an interplay between biology and the cellular mechanical environment, is required for both normal physiological function and etiology of diseases.11-13 A wide selection of tissues and cells including differentiated cells, stem cells and progenitors are regulated by mechanical signals and loading, and proper regulation of this process is essential in diseases like osteoporosis, atherosclerosis, and osteoarthritis. When bone cells do not experience adequate mechanical stimulation, bone formation ceases and a program of bone resorption is initiated. Although osteocyte's function and role in communication have been recognized by bone biologists for several decades, until recently the mechanosensing mechanisms of osteocytes were less studied for these diseases. Osteocytes have a cell body and cytoplasmic extensions, which play an important role in their function as mechanosensors. Osteocytes are for example sensitive to fluid shear stress as a result of bone loading. In addition, osteocytes have a cilium, which might also play an important role in their mechanosensory function and sends signals to regulate a variety of important phenomena in bone biology. ${ }^{14-16}$ Osteocytes may produce a single, mechanically derived signal to control
Correspondence: Nabanita S. Datta, Associate Professor of Medicine, Internal Medicine, Endocrinology, Wayne State University School of Medicine, 1107 Elliman Building, 421 East Canfield Avenue, Detroit, Michigan 48201, USA. Tel. +1.313.7454008 - Fax: +1.313.5778615.

E-mail: ndatta@med.wayne.edu

Key words: osteocytes, bone remodeling, PTH, SOST, sclerostin, Wnt.

Acknowledgements: the author was partially supported by the grants from The National Institute of Health, NIDDK087848, and funding from The Office of The Vice President for Research (OVPR), Wayne State University, during writing of this manuscript.

Conflict of interests: the author reports no conflict of interests.

Received for publication: 30 August 2012.

Revision received: 19 October 2012.

Accepted for publication: 20 October 2012.

This work is licensed under a Creative Commons Attribution NonCommercial 3.0 License (CC BYNC 3.0)

(C) Copyright N.S. Datta, 2012

Licensee PAGEPress, Italy

Endocrinology Studies 2012; 2:e8

doi:10.4081/es.2012.e8

osteoblast and bone lining cell functions and thereby regulate bone modeling and remodeling process (Figure 1).1,17,18 Thus by responding to mechanical strain osteocytes help new bone formation. Having not enough live osteocytes possibly lead to inefficiency in removing microdamage and reduced remodeling activity. ${ }^{19-22}$

Osteocytes also send signals to activate bone resorption. ${ }^{23}$ It was hypothesized that osteocyte death is responsible for signaling osteoclasts for bone resorption. Using ex vivo and in vivo animal models studies show that mechanical stimulation controls osteocyte viability thus maintaining apoptotic cell death. ${ }^{24}$ Apoptotic osteocytes co-localize with regions of bone resorption. Studying organ culture system of isolated rat calvaria Gu et al. suggested that osteocytes are negative regulator of osteoclast activity and osteocytes may play a major role in triggering local bone remodeling. ${ }^{25}$ While osteocyte apoptosis triggers the bone remodeling response to microdamage, the neighboring non-apoptotic osteocytes are the major source of pro-osteoclastogenic signals. Moreover, both the apoptotic and osteoclastsignaling osteocyte populations are localized in a spatially and temporally restricted pattern consistent with the targeted nature of this remodeling response. ${ }^{23}$ Osteocyte-ablated mice exhibit fragile bone with intracortical porosity and microfractures, osteoblastic dysfunction, 
and trabecular bone loss with microstructural deterioration. ${ }^{26}$ These mice are resistant to unloading-induced bone loss further suggesting the importance of osteocytes in mechanotransduction. Using osteocytes ablation model it was also shown that the osteocyte apoptosis is sufficient to initiate an osteoclastogenic response and that osteocytes are required for the skeletal adaptation to reduced mechanical forces. ${ }^{27-29}$ Several other studies support the concept that lack of osteocyte apoptosis do not support bone resorption.14,30-33 In human trabecular bone, mechanical forces also regulate osteocyte viability and responsible for osteoclast recruitment resulting increase in bone resorption and bone loss.23,24,32 Pro-clastogenic signals released from osteocytes such as conditioned media from apoptotic MLO-Y4 in vitro are consistent with pro-clastogenic signals observed in vivo studies implying increased osteoclastogenesis with increased osteocyte apoptosis. ${ }^{14,26,27,32-34}$ Future studies will clarify if living osteocytes continually produce antiosteoclastogenic signals that limit osteoclast recruitment or whether apoptotic osteocytes produce pro-osteoclastogenic signals.

Intracellular cross-talk within the bone ultimately makes the decision of bone formation and resorption. Identifying signaling mediators participating in the communication between osteocytes and osteoclasts and between osteocytes and osteoblasts remains to be fully explored. In regions of high bone loading, the mechano-responsive osteocytes inhibit osteoclastic bone resorption by producing signaling molecules like martix extracellular phosphoglycoprotein gene (MEPE) ${ }^{35}$ suggesting that mechanical loading of osteocytes affects osteocyte-stimulated osteoclastogenesis by involving MEPE gene. Direct cell-cell communication via gap junctions is an important phenomenon by which bone cell activities are coordinated in addition to endocrine, paracrine, and autocrine factors. Abundant gap junctions are present between osteocytic processes, between osteocytes and osteoblasts on the bone surface, and among osteoblasts.,36,37 The readers are directed to more extensive reviews on functions of osteocyte mechanobiology and pericellular mechanics which has been also discussed extensively by Jacob $e t$ $a l .4,12$ In this review the authors presented the mechanical process in bone by describing signal transduction from organ to cellular and to molecular level which finally feedbacks the biological response from molecules to organ. Starting from mechanical behavior, tissuelevel behavior to induce changes at the cellular and pericellular level, to intracellular biochemical signaling such as calcium signaling, GProtein-Mediated signaling, kinase signaling, Gap junctions, nitric oxide eicosinoid, stromal cell derived factor-1, nucleoside signaling and other cell-cell signaling mechanisms that are specific to bone and osteocyte function have been widely discussed in this review.

\section{Osteocytes in bone remodeling and parathyroid hormone/parathyroid hormone receptors signaling}

The skeleton undergoes continuous remodeling during adult life to maintain skeletal integrity, renew aging bone, and restore injuries. Initially bone remodeling was viewed to be orchestrated by bone-resorbing cells, osteoclasts and bone-forming cells, osteoblasts. It is now clear osteocytes, which are embedded into the mineralized tissue, governs profound regulatory functions on the bone remodeling process. Parathyroid hormone (PTH) exerts classical actions on bone metabolism by activating $\mathrm{PTH} / \mathrm{PTHrP}$ receptors (PTH1Rs) on target cells. Calcium, phosphorus and skeletal homeostasis are mainly regulated by PTH. Continuous exposure to PTH is associated with catabolic effects, whereas intermittent exposure to low doses of PTH is associated with anabolic effects. PTH and PTH related peptide (PTHrP) signaling in osteoblasts has been studied by us and others. ${ }^{38-45}$ Its implication in osteoporotic fractures have also been reviewed. ${ }^{46}$ Earlier studies demonstrated the presence of PTH1R on osteoblast, and that osteoblasts produce osteoclastogenic factor, receptor activator of nuclear factor $\kappa \beta$ ligand (RANKL) and macrophage colony stimulating factor (MCSF) thus activating osteoclastogenesis. ${ }^{47-}$ 52 Using genetic tools and gain-of-function and loss-of-function studies very recent report provided evidence that the major source of RANKL is osteocytes (Figure 2).53,54 New insights for osteocyte RANKL in controlling bone remodeling has been also nicely discussed. 55
Osteocytes recognition as master regulators of bone formation and localized bone remodeling led to studies revealing osteocytes importance in the PTH anabolic effects. The expression of PTH1R in osteocytes was suggested several years ago. ${ }^{56}$ This paper reported something more important though than localization, the binding of radiolabelled hormone to receptors on osteocytes after administration in vivo. The proof that osteocytes are exposed to the PTH from the circulation was a remarkable and important finding that led the later osteocyte specific PTH1R K0 studies. Targeted ablation of PTH/PTHrP receptor specifically in osteocytes under the control of Dmp-1 promoter, direct osteocyte-specific expression of transgenes, ${ }^{26,57-60}$ impairs bone structure and homeostatic calcium response in mice. ${ }^{61} \mathrm{PTH}$ potentiates the osteocytic response and this is different from osteoblast reosponse in relation to mechanotransduction pathways. ${ }^{62}$ A role for PTH1R in osteocytes and an antiapoptotic effect of vertebral osteocytes was reported using SMR1 mice. ${ }^{63}$ This antiapopototic effect by PTH was also noted either in rat distal femoral osteocytes or in vitro cultures of osteocytic cells MLO-Y4.64,65 Evidence suggest that carboxy (C)-terminal fragments of PTH, which comprise the majority of circulating PTH, do not interact with PTH1R which mediates the classical hormone actions. C-PTH exerts specific effects on calcium homeostasis and bone metabolism via a specific receptor distinct from PTH1R, known as C-terminal PTH receptors (CPTHRs). ${ }^{66,67}$ Divieti et al. reported that osteocytes expressing CPTHR may be the principal target cells for unique actions of intact PTH(1-84) and circulating PTH C-fragments. ${ }^{66,67}$ PTH receptors and apoptosis in osteocytes have been reviewed by Bringhurst. 65

Among the several signaling molecules or family of proteins in recent years those paid greatest attention for therapeutic action are SOST, Sclerostin and Wnt. In this review I will mainly focus on these molecules and their interconnections with PTH in osteocytes.

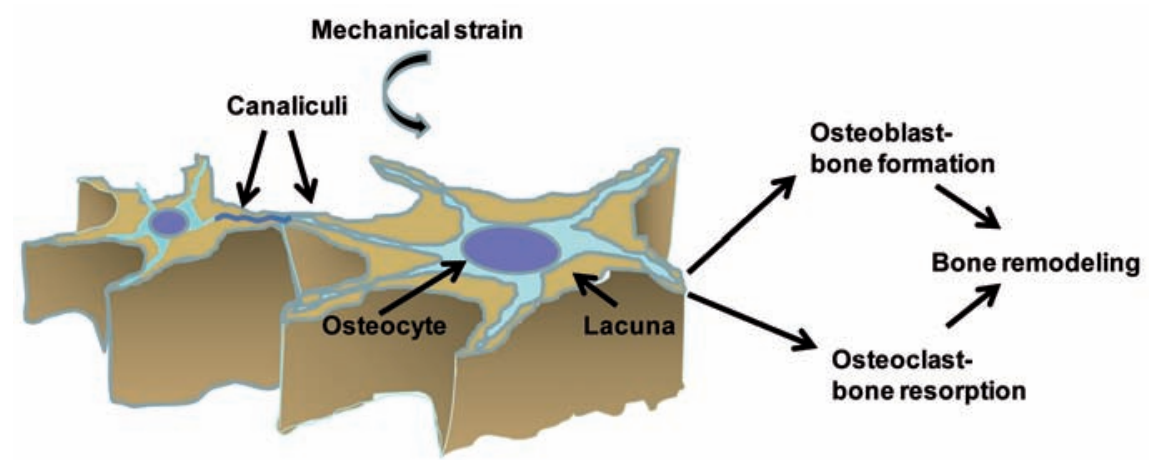

Figure 1. Osteocyte and its function. 


\section{SOST/sclerostin}

The osteocyte marker gene SOST encodes sclerostin, a secreted glycoprotein and bone formation inhibitor.68-70 Transgenic mice overexpressing human SOST shows a low bone mass phenotype and SOST knockout (KO) mice exhibit a progressive high bone mass phenotype and increased bone strength. ${ }^{71-74}$ Loss of SOST function in mice results in decreased osteocyte apoptosis.74 SOST is known to be a bone morphogenic protein (BMP) antagonist, and loss of SOST activates BMP signaling. The pro-osteoblastogenic action of BMPs and Wnts are inhibited by Sclerostin. ${ }^{75}$ Sclerostin also stimulates osteocyte support of osteoclast activity by a RANKLdependent pathway. ${ }^{76}$ Regulation of circulating sclerostin levels by sex steroids and its contribution to post menopausal osteoporosis has been a subject of investigation in recent years. ${ }^{77}$ These studies suggest a novel mechanism of hormonal control of sclerostin production in osteocyte mediated osteoblastogenesis or bone resorption. Association between sclerostin and physical activity, age, gender, body composition, and bone mineral content in healthy adults or bone density in chronic spinal cord injury has been reported. 78,79

SOST is a target gene for PTH in bone (Figure 2). ${ }^{80}$ Evidence that PTH regulates sclerostin expression comes from human and animal studies at both the cellular and molecular level. ${ }^{80-83}$ Circulating sclerostin levels may be measured in patients with overproduction or deficient PTH, namely hyperparathyroidism or hypoparathyroidism and serve as useful models to further explore the relationship between PTH and sclerostin. ${ }^{84}$ Sclerostin levels are primarily reduced in the presence of PTH. ${ }^{77,85,86}$ Continuous infusion of PTH to mice causes transcriptional suppression of SOST and reduction in sclerostin protein in vertebral bone; and decreased SOST expression in primary osteocyte cultures and in osteocytic MLOA5 cells. ${ }^{82}$ In osteocyte-containing cultures of murine calvarial cells PTH1R activation exhibit decreased SOST mRNA expression, regulates FGF-23 signaling via cAMP and Wntdependent mechanisms thus potentially modulating endocrine and auto/paracrine functions of osteocytes by. ${ }^{87}$ The elevated FGF23 expression noted in whole bones and osteocytes from DMP1-caPTHR1 mice is corrected in double transgenic mice overexpressing SOST in osteocytes. ${ }^{87}$ Classical models of PTH induced bone formation such as estrogen-deprived (OVX) rats or transgenic mice expressing a constitutively active PTHR1 specifically in osteocytes, DMP1- caPTH1R transgenic mice, or both in osteoblast and osteocyte (2.3 colcaPTH1R mice), express significantly reduced levels of SOST mRNA in calvaria or vertebral and tibial bone: ${ }^{80,82,88,89}$ and display high bone mass. ${ }^{90}$ Using SOST- deficient mice studies also suggest that changes in SOST expression are not required for anabolic effect of intermittent PTH. ${ }^{91}$ Furthermore, SOST deletion protects trabecular compartments from bone loss induced by high-dose PTH infusion.

The contribution of suppressed sclerostin level in PTH bone anabolic action has been discussed recently by Kramer et al.73,92 This review addresses how the bone anabolic responses by PTH treatment or by sclerostin inhibition overlap and diverge. Based on the striking effect of SOST inhibition in bone formation SOST has been suggested to be a potential target for therapeutic intervention in bone loss and has led to the development of sclerostin inhibitors or anti- sclerostin antibodies, thus opening new possibilities and prospects of effective anabolic therapy for bone regeneration in orthopedics and in the dental field.93-98 Sclerostin also inhibits PTH-stimulated cAMP production further suggesting neutralization of sclerostin as a potential therapy for skeletal diseases on enhancing bone formation. ${ }^{99}$

\section{Wnt}

Following the discovery of Wnt gene in 1984 the Wnt signaling pathway has been a major area of investigation and known for their roles in normal physiological processes. ${ }^{100,101}$ Since then Wnt/ $\beta$-catenin signaling pathways are being studied in bone biology because of their importance in skeletal development; bone remodeling, bone mass maintenance, regeneration and repair during a lifespan. ${ }^{102}$ Activation of Wnt pathway involves association of two membrane receptors, serum frizzled-related proteins (Fzd) and lipoprotein receptor-related proteins (LRP5/6). ${ }^{103}$ A protein complex consisting of axin, adenomatous polyposis coli, and glycogen synthase kinase 3 (GSK3) is activated following this association leading to phosphorylation of $\beta$-catenin. Accumulated $\beta$ catenin then translocates to nucleus and regulates gene transcription essential to osteoblast function. In the absence of Wnt signaling, GSK3 phosphorylates $\beta$-catenin and targets it for degradation. The Wnt signaling involves a large number of agonists and antagonists that can regulate the production and interaction of Wnt signaling molecules with the receptors on target cells, and the physiological responses of target cells. ${ }^{104}$ These antagonists bind to Wnt, Fzd, or LRP5/6 and decrease bone formation. Lack of these inhibitors activate Wnt/ $\beta$ catenin signaling and bone formation.

Osteocytes can control bone formation by modulating the Wnt signaling pathway as they represent the main source of sclerostin, the negative regulator of bone formation. Using transgenic mice engineered to maintain high levels of SOST expression it has been recently demonstrated that downregulation of SOST/sclerostin in osteocytes and activation of Wnt signaling is necessary to augment osteogenesis. ${ }^{105}$ Recent advances and discrepancies in how Wnt/Lrp5 signaling regulates osteoblasts and osteocytes has been reviewed by Monroe et al. ${ }^{102}$ This review also discusses Wnt signaling in osteoclastogenesis, new play-

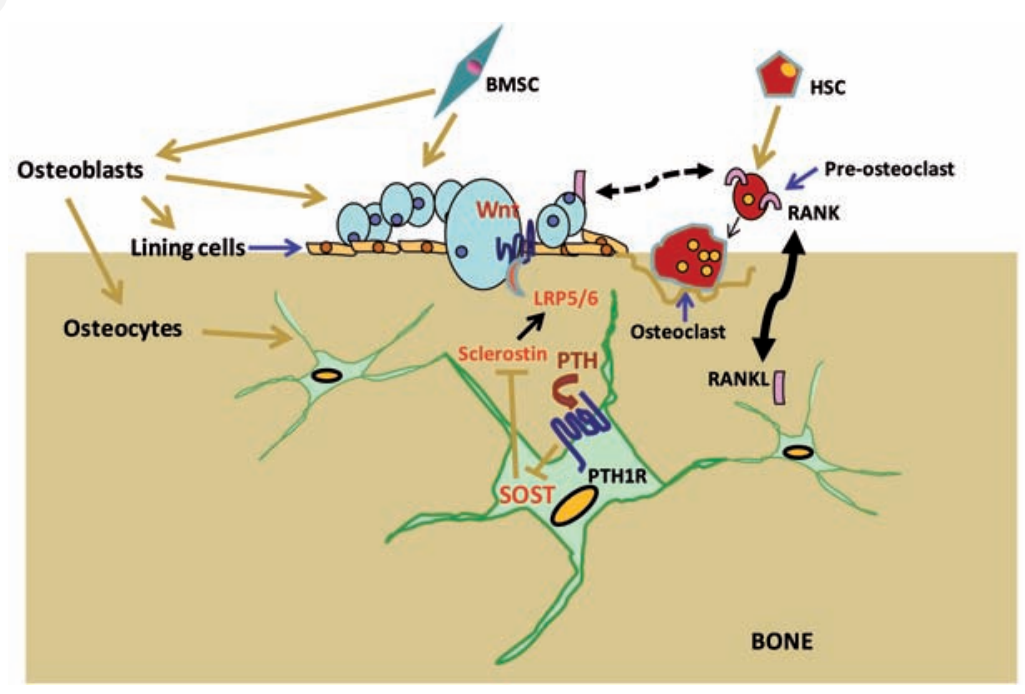

Figure 2. Parathyroid hormone (PTH) signaling in osteocytes and bone remodeling. PTH signaling in osteocytes leads to increased bone mass via suppression of SOST/Sclerostin and activating LRP5/6 Wnt signaling in osteoblasts. Osteocyte derived RANKL favors osteoclastogenesis over RANKL derived from osteoblasts. BMSC, bone marrow stromal cells; HSC, hematopoietic stem cells. 
ers in this pathways that have important roles in bone development, and progresses made in translating basic studies of Wnt pathways/antagonists to clinical therapeutics and diagnostics. Since Wnt signaling pathway plays a central role in bone development, homeostasis and in promoting bone growth Wnt factors could be used to stimulate bone healing. ${ }^{106}$ Using mouse strain Axin2 ${ }^{\text {LacZLacZ }}$ in which the cellular response to Wnt is increased it was found that bone healing after injury is accelerated in these mice, implying increased proliferation and earlier differentiation of skeletal stem and progenitor cells. It was further shown that purified Wnt3a in liposomal vesicles at sites with skeletal defects stimulates the proliferation of skeletal progenitor cells and accelerates their differentiation into osteoblasts resulting in faster bone regeneration. These studies also suggested that $W n t$ signaling and protein-based approach may have widespread applications in regenerative medicine. It is now clear that the Wnt signaling pathway is central to regulation of both skeletal modeling and remodeling and potential role for therapies targeting DKK1, LRP5, and serotonin in the treatment of osteoporosis is gaining much attention. ${ }^{97,107}$

Intermittent PTH promotes osteoblast differentiation partially by its ability to exit from the cell cycle, ${ }^{38-40}$ activating Wnt signaling in osteoblasts, ${ }^{108}$ and suppressing expression of the Wnt antagonist sclerostin in osteocytes as observed in mice and in human. ${ }^{92,99,109}$

PTH suppression of sclerostin in osteocytes increases the availability of LRP6 in PTH signaling via positive feedback mechanism (Figure 2). ${ }^{99}$ It was also suggested that PTH1R signaling in osteocytes increases bone mass and the rate of bone remodeling through LRP5dependent and -independent mechanisms, respectively. ${ }^{90}$ Multiple signaling molecules including sclerostin are utilized by PTH which cross talk and work together to modulate Wnt/3-Catenin signaling pathway and promote bone formation.110 Extensive studies on the mechanisms of action of PTH on osteocytes will help to identify new pathways that regulate bone formation leading to possible therapeutic interventions.

\section{Concluding remarks}

Given the central role of osteocytes in bone remodeling it is not surprising that the molecules regulated by osteocyte signaling figure prominently in physiological and pathophysiological pathways of bone. What is surprising is the fact that osteocytes seem to play such an essential role in bone remodeling despite the fact that the signaling molecules governed by these cells are also regulated by bone forming osteoblasts and/or bone resorbing osteoclasts. This observation highlights the importance of osteocytes in serving as a governor general in critical signaling network of bone homeostasis.

New and exciting findings are now emerging, but many questions remain unanswered: i) Does osteocyte signaling or mutational status of signaling molecules/receptors correlate with disease progression and survival? ii) Can we utilize current studies to predict patient outcome? iii) What are the important signaling pathways in osteocytes activated or deactivated in response to different bone diseases? iv) What are the mechanisms that regulate osteocyte activity in cancer metastasis to bone? v) Are osteocytes viable therapeutic target in bone metastasis? Future progress in these areas may open up new avenues of therapeutic interventions for metabolic bone diseases including osteoporosis and cancer metastasis to bone.

\section{References}

1. Bonewald LF. The amazing osteocyte. J Bone Miner Res 2011;26:229-38.

2. Bonewald L. The holy grail of high bone mass. Nat Med 2011;17:657-8.

3. Parfitt AM. The cellular basis of bone turnover and bone loss: a rebuttal of the osteocytic resorption-bone flow theory. Clin Orthop Relat Res 1977:236-47.

4. Qing H, Bonewald LF. Osteocyte remodeling of the perilacunar and pericanalicular matrix. Int J Oral Sci 2009;1:59-65.

5. Dunstan CR, Somers NM, Evans RA. Osteocyte death and hip fracture. Calcif Tissue Int 1993;53Suppl1:S113-7.

6. Frost HM. In vivo osteocyte death. J Bone Joint Surg Am 1960;42-A:138-43.

7. Donahue HJ. Gap junctions and biophysical regulation of bone cell differentiation. Bone. 2000;26:417-22.

8. Qing H, Ardeshirpour L, Pajevic PD, et al. Demonstration of osteocytic perilacunar/ canalicular remodeling in mice during lactation. J Bone Miner Res 2012;27:1018-29.

9. Dallas SL, Bonewald LF. Dynamics of the transition from osteoblast to osteocyte. Ann N Y Acad Sci 2010;1192:437-43.

10. Delgado-Calle J, Sanudo C, Bolado A, et al. DNA methylation contributes to the regulation of sclerostin expression in human osteocytes. J Bone Miner Res 2012;27:92637.

11. Jacobs CR. The mechanobiology of cancellous bone structural adaptation. J Rehabil Res Dev 2000;37:209-16.

12. Jacobs CR, Temiyasathit S, Castillo AB. Osteocyte mechanobiology and pericellular mechanics. Annu Rev Biomed Eng 2010;12:369-400.
13. Stoltz JF, Wang X. From biomechanics to mechanobiology. Biorheology 2002;39:510.

14. Zhao S, Zhang YK, Harris S, et al. MLO-Y4 osteocyte-like cells support osteoclast formation and activation. J Bone Miner Res 2002;17:2068-79.

15. Bonewald LF. Establishment and characterization of an osteocyte-like cell line, MLO-Y4. J Bone Miner Metab 1999;17:615 .

16. Hoey DA, Chen JC, Jacobs CR. The primary cilium as a novel extracellular sensor in bone. Front Endocrinol (Lausanne) 2012;3:75.

17. Martin RB. Toward a unifying theory of bone remodeling. Bone 2000;26:1-6.

18. Bonewald LF. Osteocyte biology: its implications for osteoporosis. J Musculoskelet Neuronal Interact 2004;4:101-4.

19. Mori S, Harruff R, Ambrosius W, Burr DB. Trabecular bone volume and microdamage accumulation in the femoral heads of women with and without femoral neck fractures. Bone 1997;21:521-6.

20. Kamijou T, Nakajima T, Ozawa H. Effects of osteocytes on osteoinduction in the autogenous rib graft in the rat mandible. Bone 1994;15:629-37.

21. Marotti G. The structure of bone tissues and the cellular control of their deposition. Ital J Anat Embryol 1996;101:25-79.

22. Marotti G, Farneti D, Remaggi F, Tartari F. Morphometric investigation on osteocytes in human auditory ossicles. Ann Anat 1998;180:449-53.

23. Kennedy OD, Herman BC, Laudier DM, et al. Activation of resorption in fatigueloaded bone involves both apoptosis and active pro-osteoclastogenic signaling by distinct osteocyte populations. Bone 2012;50:1115-22.

24. Mann V, Huber C, Kogianni G, et al. The influence of mechanical stimulation on osteocyte apoptosis and bone viability in human trabecular bone. J Musculoskelet Neuronal Interact 2006;6:408-17.

25. Gu G, Mulari M, Peng Z, et al. Death of osteocytes turns off the inhibition of osteoclasts and triggers local bone resorption. Biochem Biophys Res Commun 2005;335:1095-101.

26. Tatsumi S, Ishii $\mathrm{K}$, Amizuka $\mathrm{N}$, et al. Targeted ablation of osteocytes induces osteoporosis with defective mechanotransduction. Cell Metab 2007;5:464-75.

27. Aguirre JI, Plotkin LI, Stewart SA, et al. Osteocyte apoptosis is induced by weightlessness in mice and precedes osteoclast recruitment and bone loss. J Bone Miner Res 2006;21:605-15.

28. Ikeda K. Osteocytes in the pathogenesis of osteoporosis. Geriatr Gerontol Int 2008; 8:213-7. 
29. Bellido T. Antagonistic interplay between mechanical forces and glucocorticoids in bone: a tale of kinases. $\mathrm{J}$ Cell Biochem 2010;111:1-6.

30. Cardoso L, Herman BC, Verborgt 0, et al. Osteocyte apoptosis controls activation of intracortical resorption in response to bone fatigue. J Bone Miner Res 2009;24: 597-605.

31. Herman BC, Cardoso L, Majeska RJ, et al. Activation of bone remodeling after fatigue: differential response to linear microcracks and diffuse damage. Bone 2010;47:766-72.

32. Al-Dujaili SA, Lau E, Al-Dujaili $\mathrm{H}$, et al. Apoptotic osteocytes regulate osteoclast precursor recruitment and differentiation in vitro. J Cell Biochem 2011;112:2412-23.

33. Shandala T, Shen Ng Y, Hopwood B, et al. The role of osteocyte apoptosis in cancer chemotherapy-induced bone loss. J Cell Physiol 2012;227:2889-97.

34. Giuliani N, Ferretti M, Bolzoni M, et al. Increased osteocyte death in multiple myeloma patients: role in myelomainduced osteoclast formation. Leukemia 2012. Epub 2012/02/01.

35. Kulkarni RN, Bakker AD, Everts V, KleinNulend J. Inhibition of osteoclastogenesis by mechanically loaded osteocytes: involvement of MEPE. Calcif Tissue Int 2010;87:461-8.

36. Doty SB. Morphological evidence of gap junctions between bone cells. Calcif Tissue Int 1981;33:509-12.

37. Palumbo C, Palazzini S, Marotti G. Morphological study of intercellular junctions during osteocyte differentiation. Bone 1990;11:401-6.

38. Datta NS, Chen C, Berry JE, McCauley LK. PTHrP signaling targets cyclin D1 and induces osteoblastic cell growth arrest. J Bone Miner Res 2005;20:1051-64.

39. Datta NS, Pettway GJ, Chen C, et al. Cyclin D1 as a target for the proliferative effects of PTH and PTHrP in early osteoblastic cells. J Bone Miner Res 2007;22:951-64.

40. Datta NS, Kolailat R, Fite A, et al. Distinct roles for mitogen-activated protein kinase phosphatase-1 (MKP-1) and ERK-MAPK in PTH1R signaling during osteoblast proliferation and differentiation. Cell Signal 2010;22:457-66.

41. Datta NS, Samra TA, Mahalingam CD, et al. Role of PTH1R internalization in osteoblasts and bone mass using a phosphorylation-deficient knock-in mouse model. J Endocrinol 2010;207:355-65.

42. Mahalingam CD, Datta T, Patil RV, et al. Mitogen activated protein kinase phosphatase-1 regulates bone mass, osteoblast gene expression, and responsiveness to parathyroid hormone. J Endocrinol 2011; 211:145-56.
43. Datta NS, Samra TA, Abou-Samra AB. Parathyroid hormone (PTH) induces bone formation in phosphorylation deficient PTHR1 knock-in mice. Am J Physiol Endocrinol Metab 2012;302:E1183-E8.

44. Jilka RL. Molecular and cellular mechanisms of the anabolic effect of intermittent PTH. Bone 2007;40:1434-46.

45. Datta NS, Abou-Samra AB. PTH and PTHrP signaling in osteoblasts. Cell Signal 2009; 21:1245-54.

46. Datta NS. Osteoporotic fracture and parathyroid hormone. World J Orthop 2011;2:67-74.

47. Rodan GA, Martin TJ. Role of osteoblasts in hormonal control of bone resorption-a hypothesis. Calcif Tissue Int 1981;33:34951.

48. Takahashi N, Akatsu T, Udagawa N, et al. Osteoblastic cells are involved in osteoclast formation. Endocrinology 1988;123: 2600-2.

49. Udagawa N, Takahashi N, Akatsu T, et al. The bone marrow-derived stromal cell lines MC3T3-G2/PA6 and ST2 support osteoclast-like cell differentiation in cocultures with mouse spleen cells. Endocrinology 1989;125:1805-13.

50. Lacey DL, Timms E, Tan HL, et al. Osteoprotegerin ligand is a cytokine that regulates osteoclast differentiation and activation. Cell 1998;93:165-76.

51. Yasuda H, Shima N, Nakagawa N, et al. Osteoclast differentiation factor is a ligand for osteoprotegerin/osteoclastogenesisinhibitory factor and is identical to TRANCE/RANKL. Proc Natl Acad Sci U S A 1998;95:3597-602.

52. Yoshida H, Hayashi S, Kunisada T, et al. The murine mutation osteopetrosis is in the coding region of the macrophage colony stimulating factor gene. Nature 1990;345:442-4.

53. Nakashima T, Hayashi M, Fukunaga T, et al. Evidence for osteocyte regulation of bone homeostasis through RANKL expression. Nat Med 2011;17:1231-4.

54. Xiong J, Onal M, Jilka RL, et al. Matrixembedded cells control osteoclast formation. Nat Med 2011;17:1235-41.

55. Xiong J, O'Brien CA. Osteocyte RANKL: new insights into the control of bone remodeling. J Bone Miner Res 2012;27: 499-505.

56. Fermor B, Skerry TM. PTH/PTHrP receptor expression on osteoblasts and osteocytes but not resorbing bone surfaces in growing rats. J Bone Miner Res 1995;10:193543.

57. Kalajzic I, Braut A, Guo D, et al. Dentin matrix protein 1 expression during osteoblastic differentiation, generation of an osteocyte GFP-transgene. Bone 2004; 35:74-82.
58. Yang W, Lu Y, Kalajzic I, Guo D, et al. Dentin matrix protein 1 gene cis-regulation: use in osteocytes to characterize local responses to mechanical loading in vitro and in vivo. J Biol Chem 2005;280:2068090 .

59. Feng JQ, Ward LM, Liu S, et al. Loss of DMP1 causes rickets and osteomalacia and identifies a role for osteocytes in mineral metabolism. Nat Genet 2006;38:13105.

60. Lu Y, Xie Y, Zhang S, et al. DMP1-targeted Cre expression in odontoblasts and osteocytes. J Dent Res 2007;86:320-5.

61. Powell WF Jr., Barry KJ, Tulum I, et al. Targeted ablation of the PTH/PTHrP receptor in osteocytes impairs bone structure and homeostatic calcemic responses. J Endocrinol 2011;209:21-32.

62. Mikuni-Takagaki Y, Naruse K, Azuma Y, Miyauchi A. The role of calcium channels in osteocyte function. J Musculoskelet Neuronal Interact 2002;2:252-5.

63. Jilka RL, Weinstein RS, Bellido T, et al. Increased bone formation by prevention of osteoblast apoptosis with parathyroid hormone. J Clin Invest 1999;104:439-46.

64. Stanislaus D, Yang X, Liang JD, et al. In vivo regulation of apoptosis in metaphyseal trabecular bone of young rats by synthetic human parathyroid hormone (1-34) fragment. Bone 2000;27:209-18.

65. Bringhurst FR. PTH receptors and apoptosis in osteocytes. J Musculoskelet Neuronal Interact 2002;2:245-51.

66. Divieti P, Inomata N, Chapin $\mathrm{K}$, et al. Receptors for the carboxyl-terminal region of pth(1-84) are highly expressed in osteocytic cells. Endocrinology 2001;142:916-25.

67. Selim AA, Mahon M, Juppner H, et al. Role of calcium channels in carboxyl-terminal parathyroid hormone receptor signaling. Am J Physiol Cell Physiol 2006;291:C11421.

68. Poole KE, van Bezooijen RL, Loveridge N, et al. Sclerostin is a delayed secreted product of osteocytes that inhibits bone formation. FASEB J 2005;19:1842-4.

69. van Bezooijen RL, Roelen BA, Visser A, et al. Sclerostin is an osteocyte-expressed negative regulator of bone formation, but not a classical BMP antagonist. J EXP Med 2004;199:805-14.

70. van Bezooijen RL, ten Dijke P, Papapoulos SE, Lowik CW. SOST/sclerostin, an osteocyte-derived negative regulator of bone formation. Cytokine Growth Factor Rev 2005;16:319-27.

71. Loots GG, Kneissel M, Keller H, et al. Genomic deletion of a long-range bone enhancer misregulates sclerostin in Van Buchem disease. Genome Res 2005;15: 928-35.

72. Li X, Ominsky MS, Niu QT, et al. Targeted 
deletion of the sclerostin gene in mice results in increased bone formation and bone strength. J Bone Miner Res 2008;23: 860-9.

73. Kramer I, Loots GG, Studer A, et al. Parathyroid hormone (PTH)-induced bone gain is blunted in SOST overexpressing and deficient mice. J Bone Miner Res 2010;25:178-89.

74. Lin C, Jiang X, Dai Z, et al. Sclerostin mediates bone response to mechanical unloading through antagonizing Wnt/betacatenin signaling. J Bone Miner Res 2009; 24:1651-61.

75. Winkler DG, Sutherland MS, Ojala E, et al. Sclerostin inhibition of Wnt-3a-induced C3H10T1/2 cell differentiation is indirect and mediated by bone morphogenetic proteins. J Biol Chem 2005;280:2498-502.

76. Wijenayaka AR, Kogawa M, Lim HP, et al Sclerostin stimulates osteocyte support of osteoclast activity by a RANKL-dependent pathway. PLoS One 2011;6:e25900.

77. Modder UI, Clowes JA, Hoey K, et al. Regulation of circulating sclerostin levels by sex steroids in women and in men. $\mathrm{J}$ Bone Miner Res 2011;26:27-34.

78. Morse LR, Sudhakar S, Danilack V, et al. Association between sclerostin and bone density in chronic spinal cord injury. J Bone Miner Res 2012;27:352-9.

79. Amrein K, Amrein S, Drexler C, et al. Sclerostin and its association with physical activity, age, gender, body composition, and bone mineral content in healthy adults. J Clin Endocrinol Metab 2012;97: 148-54.

80. Keller H, Kneissel M. SOST is a target gene for PTH in bone. Bone 2005;37:14858.

81. Silvestrini G, Ballanti P, Leopizzi M, et al. Effects of intermittent parathyroid hormone (PTH) administration on SOST mRNA and protein in rat bone. $\mathrm{J} \mathrm{Mol} \mathrm{Histol}$ 2007;38:261-9.

82. Bellido T, Ali AA, Gubrij I, et al. Chronic elevation of parathyroid hormone in mice reduces expression of sclerostin by osteocytes: a novel mechanism for hormonal control of osteoblastogenesis. Endocrinology 2005;146:4577-83.

83. Drake MT, Srinivasan B, Modder UI, et al. Effects of parathyroid hormone treatment on circulating sclerostin levels in postmenopausal women. J Clin Endocrinol Metab 2010;95:5056-62.

84. Gaudio A, Pennisi P, Bratengeier C, et al.
Increased sclerostin serum levels associated with bone formation and resorption markers in patients with immobilizationinduced bone loss. J Clin Endocrinol Metab 2010;95:2248-53.

85. Mirza FS, Padhi ID, Raisz LG, Lorenzo JA. Serum sclerostin levels negatively correlate with parathyroid hormone levels and free estrogen index in postmenopausal women. J Clin Endocrinol Metab 2010;95: 1991-7.

86. van Lierop AH, Witteveen JE, Hamdy NA, Papapoulos SE. Patients with primary hyperparathyroidism have lower circulating sclerostin levels than euparathyroid controls. Eur J Endocrinol 2010;163:833-7.

87. Rhee Y, Bivi N, Farrow E, et al. Parathyroid hormone receptor signaling in osteocytes increases the expression of fibroblast growth factor-23 in vitro and in vivo. Bone 2011;49:636-43.

88. Calvi LM, Sims NA, Hunzelman JL, et al. Activated parathyroid hormone/parathyroid hormone-related protein receptor in osteoblastic cells differentially affects cortical and trabecular bone. J Clin Invest 2001;107:277-86.

89. Bellido T. Downregulation of SOST/sclerostin by PTH: a novel mechanism of hormonal control of bone formation mediated by osteocytes. J Musculoskelet Neuronal Interact 2006;6:358-9.

90. O'Brien CA, Plotkin LI, Galli C, et al. Control of bone mass and remodeling by PTH receptor signaling in osteocytes. PLoS One 2008;3:e2942.

91. Robling AG, Kedlaya R, Ellis SN, et al. Anabolic and catabolic regimens of human parathyroid hormone 1-34 elicit bone- and envelope-specific attenuation of skeletal effects in Sost-deficient mice. Endocrinology 2011;152:2963-75.

92. Kramer I, Keller H, Leupin 0, Kneissel M. Does osteocytic SOST suppression mediate PTH bone anabolism? Trends Endocrinol Metab 2010;21:237-44.

93. Rawadi G, Roman-Roman S. Wnt signalling pathway: a new target for the treatment of osteoporosis. Expert Opin Ther Targets 2005;9:1063-77.

94. Baron R, Rawadi G. Wnt signaling and the regulation of bone mass. Curr Osteoporos Rep 2007;5:73-80.

95. Shahnazari M, Yao W, Corr M, Lane NE. Targeting the Wnt signaling pathway to augment bone formation. Curr Osteoporos Rep 2008;6:142-8.
96. Deal C. Potential new drug targets for osteoporosis. Nat Clin Pract Rheumatol 2009;5:20-7.

97. Hoeppner LH, Secreto FJ, Westendorf JJ. Wnt signaling as a therapeutic target for bone diseases. Expert Opin Ther Targets 2009;13:485-96

98. Galli C, Passeri G, Macaluso GM Osteocytes and WNT: the mechanical control of bone formation. J Dent Res 2010; 89:331-43.

99. Shi C, Li J, Wang W, et al. Antagonists of LRP6 regulate PTH-induced cAMP generation. Ann N Y Acad Sci 2011;1237:39-46.

100.Nusse R, van Ooyen A, Cox D, et al. Mode of proviral activation of a putative mammary oncogene (int-1) on mouse chromosome 15. Nature 1984;307:131-6.

101.Yang Y. Wnt signaling in development and disease. Cell Biosci 2012;2:14.

102.Monroe DG, McGee-Lawrence ME, Oursler MJ, Westendorf JJ. Update on Wnt signaling in bone cell biology and bone disease. Gene 2012;492:1-18.

103.Kikuchi A, Yamamoto H, Kishida S. Multiplicity of the interactions of Wnt proteins and their receptors. Cell Signal 2007;19:659-71.

104.MacDonald BT, Tamai K, He X. Wnt/betacatenin signaling: components, mechanisms, and diseases. Dev Cell 2009;17:926.

105.Tu X, Rhee Y, Condon KW, et al. Sost downregulation and local Wnt signaling are required for the osteogenic response to mechanical loading. Bone 2012;50:209-17.

106. Minear S, Leucht P, Jiang J, et al. Wnt proteins promote bone regeneration. Sci Transl Med 2010;2:29-30.

107.Zhang W, Drake MT. Potential role for therapies targeting DKK1, LRP5, and serotonin in the treatment of osteoporosis. Curr Osteoporos Rep 2012;10:93-100.

108.Tian Y, Xu Y, Fu Q, He M. Parathyroid hormone regulates osteoblast differentiation in a Wnt/beta-catenin-dependent manner. Mol Cell Biochem 2011;355:211-6.

109.Costa AG, Cremers S, Rubin MR, et al. Circulating sclerostin in disorders of parathyroid gland function. J Clin Endocrinol Metab 2011;96:3804-10.

110.Fei Y, Hurley MM. Role of fibroblast growth factor 2 and Wnt signaling in anabolic effects of parathyroid hormone on bone formation. J Cell Physiol 2012;227:353945. 